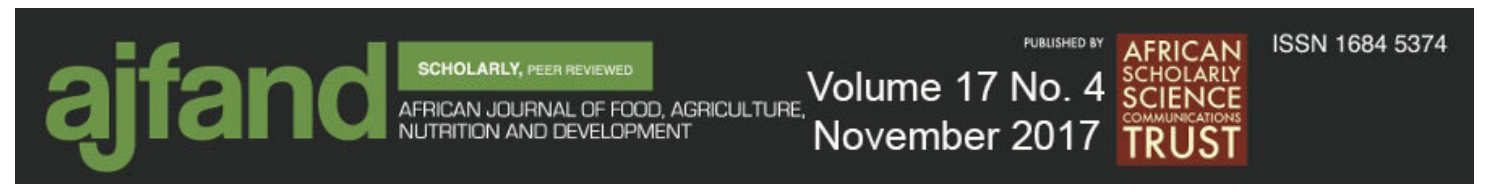

\title{
FACTORS ASSOCIATED WITH ANAEMIA STATUS AND HAEMOGLOBIN CONCENTRATIONS IN INFANTS 6-11 MONTHS IN MBALA DISTRICT, NORTHERN PROVINCE, ZAMBIA
}

\author{
Daly $\mathbf{Z}^{1}$, Northrup-Lyons $\mathbf{M}^{2}$, Brunet $\mathrm{D}^{3}$, \\ Aongola $\mathrm{A}^{4}$, Green $\mathrm{TJ}^{5,6}$, and $\mathrm{J}$ McLean ${ }^{1^{*}}$
}

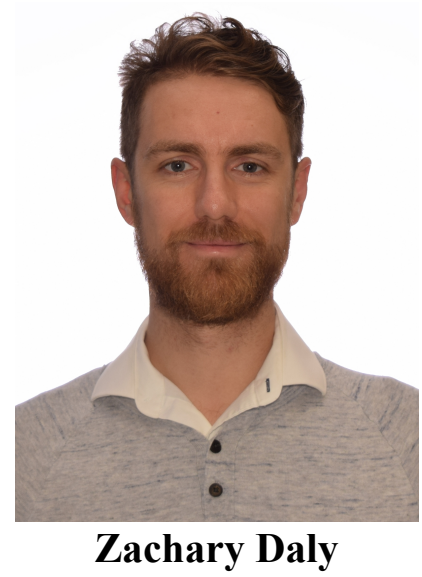

*Corresponding author email: judy.mclean@ubc.ca

${ }^{\mathbf{1}}$ Food, Nutrition, and Health, University of British Columbia, Vancouver, Canada

${ }^{2}$ Micronutrient Project, University of British Columbia, Vancouver, Canada

${ }^{3}$ UNICEF Zambia, Lusaka, Zambia

${ }^{4}$ Ministry of Health, Lusaka, Zambia

${ }^{5}$ South Australian Health and Medical Research Institute, Adelaide, Australia

${ }^{6}$ Discipline of Paediatrics and Reproductive Health, University of Adelaide, Adelaide, Australia 


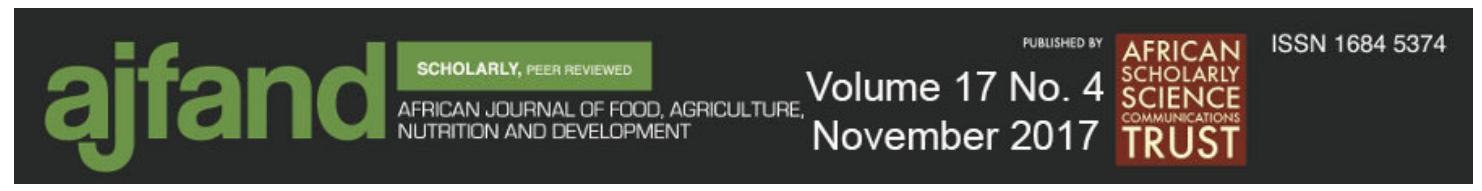

\begin{abstract}
This paper presents information on the factors associated with anaemia status and haemoglobin concentrations in infants 6-11 months old in Mbala District, Northern Province, Zambia. The information is drawn from the baseline data collection of a study involving 631 caregiver-child pairs. The data presented here includes anthropometry, malaria parasitism, concentrations of haemoglobin $(\mathrm{Hb})$ and serum transferrin receptor (STfR), and information on household characteristics, infant and young child feeding (IYCF) practices, and morbidity, gathered via questionnaire. It was found that $57 \%$ of the sample was anaemic $(\mathrm{Hb}<110 \mathrm{~g} / \mathrm{L}), 93 \%$ was iron deficient (STfR $>8.3 \mathrm{mg} / \mathrm{L}$ ) and 53\% had iron deficiency anaemia. Overall, 30\% of the infants in the sample were stunted, $2 \%$ were wasted, and $16 \%$ were underweight, and $22 \%, 56 \%$ and $18 \%$ of infants were meeting minimum dietary diversity, minimum meal frequency, and minimum acceptable diet, respectively, and $28 \%$ consumed iron rich foods in the previous 24 hours. Infants who had achieved a minimum dietary diversity score $(\mathrm{OR}=0.44 ; 95 \% \mathrm{CI}: 0.30-0.65)$, who had consumed iron rich foods in the previous 24 hours $(\mathrm{OR}=0.56 ; 95 \% \mathrm{CI}: 0.40-0.80)$, or were female $(\mathrm{OR}=0.69 ; 95 \% \mathrm{CI}: 0.50-0.94)$, had significantly lower risks of being anaemic. Infants that tested positive for malaria parasitism $(\mathrm{OR}=4.33$; 95\% CI: $2.16-$ $8.70)$, as well as those having a fever (OR $=1.88$; 95\% CI: $1.36-2.59)$ in the previous two weeks had significantly greater odds of being anaemic. Despite these associations, whether or not a child slept under a mosquito net was not related to their risk of being anaemic. Infants in households that treated their water to make it safer to consume had average haemoglobin concentrations $3 \mathrm{~g} / \mathrm{L}$ higher than those who did not $(\mathrm{p}=0.021)$, but this did not translate into a significant difference in the odds of being anaemic. These results shed light on the specific causes of anaemia in the Zambian context, and illustrate the importance of improving dietary quality, specifically the intake of iron, and reducing the prevalence of diseases including malaria in order to reduce the prevalence of anaemia.
\end{abstract}

Key words: Nutritional status, Zambia, anaemia, iron deficiency, malaria, serum transferrin receptor, haemoglobin 


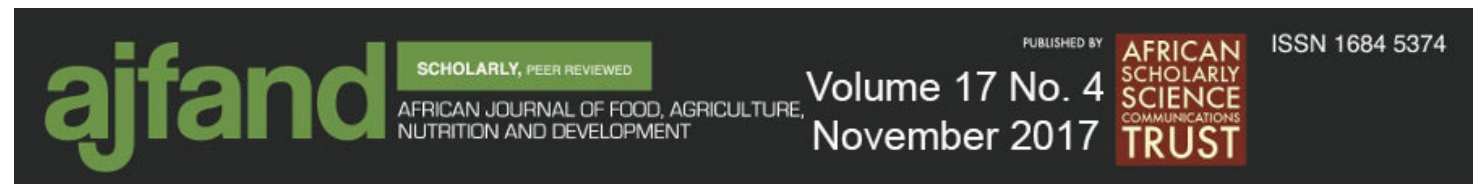

\section{INTRODUCTION}

Anaemia is a preventable condition of global significance, particularly among infants and young children 6-23 months of age [1]. Illustrating the scale of the problem, the most recent estimates of anaemia prevalence from the WHO suggest that globally $25 \%$ of all people suffer from anaemia, corresponding to over 1.6 billion people [2]. The global prevalence among preschool children $(0-59$ months $)$ is the highest of any age group (47\%), representing 293 million individuals. Within Africa, the prevalence of anaemia among preschool children was estimated to be $65 \%$, representing 93.2 million children [2].

Rates of childhood anaemia are high within Zambia. According to the 2012 National Malaria Indicator Survey, 55\% of children under five were anaemic, defined as haemoglobin $(\mathrm{Hb})$ concentrations of less than $110 \mathrm{~g} / \mathrm{L}$ [3]. The prevalence of anaemia was even higher in infants under 12 months of age (70\%) [3]. this is well above the $40 \%$ cut-off which the World Health Organization (WHO) uses to designate a "severe public health problem" [2]. The consequences of anaemia include loss of appetite, fatigue, poor regulation of temperature, and gastrointestinal disturbances [2, 4]. If the anaemia is the result of iron deficiency, consequences may include irreversible, impaired, cognitive development [4].

Previous research on the etiology of anaemia in Zambia has suggested a link to malaria $[5,6,7,8]$, and possibly helminths [6]. Research on the importance of iron deficiency, possibly as a result of the plant based diet, is mixed and may in fact differ from region to region; comparison is complicated by differences in how iron deficiency was assessed in different studies $[7,9,10,11]$. There is evidence that there may be an association between indicators of socio-economic status and anaemia [9], although this has not been consistent [6]. There are haemoglobinopathies in Zambia, such as sickle cell trait, but there is no nationally representative data on their prevalence, making it difficult to state their importance in understanding anaemia in the country [12].

This paper will add to the literature by looking at potential factors associated with anaemia in the Northern Province of Zambia, as well as on the nutritional status of a young cohort: infants aged 6 to 11 months. The results should prove useful in understanding anaemia in Zambia. Most importantly, the results will be useful in the design of future interventions aimed at improving nutrition in Zambia and elsewhere.

\section{MATERIALS AND METHODS}

This paper is based on analysis of baseline data from a larger study examining micronutrient powders (MNP) in Mbala District, Northern Province.

\section{Sample and study design}

Data collection took place during the dry season, in June 2013. The study made use of a convenience sample, as a random sample was deemed not feasible: local partners who had conducted research in the region indicated that using a random sample would 
cause suspicion in the communities, as it would create unease as to why only some individuals were chosen to participate in the research over others and there was already some suspicion in the communities around research involving blood due to local beliefs. Sampling was based on the Catchment Areas used by the local health system. Of the 26 Catchment Areas in Mbala District, nine were selected: Kaka, Kamuzwazi, Kawimbe, Mambwe Mission, Mbala Urban, Mpande, Nondo, Senga and Tulemane.

Catchment Areas were selected to ensure a mix of both rural and more urbanized areas, as well as a mix of areas close to and distant from the main paved road. Catchment areas are further divided into zones and within each of the selected Catchment Areas, five to six zones were chosen, for a total of 52 zones. Zones not accessible during the rainy season and with very low populations were excluded. A total goal for recruitment was set at least 620 caregiver-child pairs, in order to provide sufficient statistical power to the larger micronutrient powder study that this baseline data collection was a part of. Of the 52 zones or sites, four were based at local health centres and the other 48 were located at local schools, which served as points of contact for the health system. Before going to each zone to start recruitment, community mobilisation and sensitization teams from The National Food and Nutrition Commission of Zambia travelled beforehand, in order to meet with community members and community leaders, and provide information about the project and answer questions. These teams also provided information about where and what dates the data collection team would be arriving in the community, either at the health centre, or at a local school as appropriate, thus ensuring that caregiver-child dyads would show up on the day of recruitment. On the day of recruitment for each site, the team went and met again with local community leaders before starting recruitment at the previously agreed location. Caregiver-child dyads were interviewed on a first-come, first-interviewed basis, after having ensured that they met the inclusion criteria and providing consent, as described below.

In the end, a total of 631 eligible caregiver-infant pairs were recruited for the study. Haemoglobin measurements were obtained from 631 infants. Thick blood smears were obtained from 625 infants and serum transferrin receptor was assessed for 620 individuals. Valid results were obtained from 630 individuals for stunting, from 628 individuals for wasting, and 629 individuals for underweight. The final distribution of individuals between the catchment areas was as follows: 80 in Nondo, 79 in Kamuzwazi, 78 in Kaka, 71 in Mbala Urban, 70 in Mpande, 65 in Mambwe Mission, 65 in Senga, 63 in Tulemane and 60 in Kawimbe. Of the originally chosen 52 zones, one zone was in the end not used for recruitment due to logistical issues, giving a final count of 51 zones.

The inclusion criteria were:

i. $\quad$ Infants aged 6-11 months;

ii. Infants residing within one of the nine project Catchment Areas, and who planned on remaining in the same household for twelve months; 


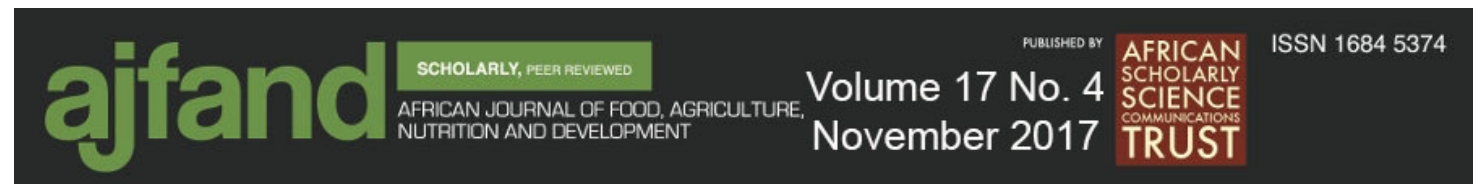

iii. Parent/guardian willingness to give consent for the child's participation in the study.

The exclusion criteria were:

i. Severe acute malnutrition (weight-for-height Z-score $<-3$ standard deviations $(\mathrm{SD}), \mathrm{MUAC}<11.5 \mathrm{~cm}$, and/or the presence of bilateral oedema) [13];

ii. Severe anaemia (haemoglobin concentration $<70 \mathrm{~g} / \mathrm{L})[14]$;

iii. HIV positive infants, as determined by their Zambian 'Under 5 Child Card', any other documentation, or during the questionnaire.

Infants who were excluded from the study as a result of severe acute malnutrition (SAM) or severe anaemia were referred to the nearest hospital or clinic for treatment.

\section{Ethical considerations}

Ethical approval was obtained from the Tropical Disease Research Centre (TDRC) Ethics Review Committee in Zambia, approval number TRC/C4/05/2013, and the University of British Columbia (UBC) Clinical Research Ethics Board, approval number H13-00261. The study was registered with ClinicalTrials.gov as NCT01878734. Participation in the study was voluntary: participants could withdraw at any time without consequences, and informed consent was obtained from caregivers.

\section{Household questionnaires}

A questionnaire was administered to each caregiver by a Zambian enumerator. The questionnaire contained seven modules: 'Anthropometry, Oedema \& Biochemical'; 'Household Information'; 'Drinking Water, Hygiene \& Sanitation'; 'Early Childhood Development'; 'Child Health \& Health Seeking Behaviour'; 'Caregiver Knowledge of Infant and Young Child Feeding Practices \& Anaemia', and 'Infant and Young Child Feeding (IYCF) Practices \& Food Consumption'. This information was used to calculate the standard IYCF indicators of minimum meal frequency, minimum dietary diversity, and minimum acceptable diet, using WHO definitions [15].

\section{Anthropometric assessment}

The length, weight, age and mid upper-arm circumference of each child was obtained to calculate z-scores for weight-for-age (underweight), height-for-age (stunting) and weight-for-height (wasting), as per the WHO Growth Standards [16]. Length was measured using a recumbent length board [17]. Weight was measured using a battery powered digital flat scale manufactured by SECA ${ }^{\mathrm{TM}}$, which was calibrated using known weights [17]. Mid upper-arm circumference (MUAC) was measured using a standard MUAC tape [17]. 


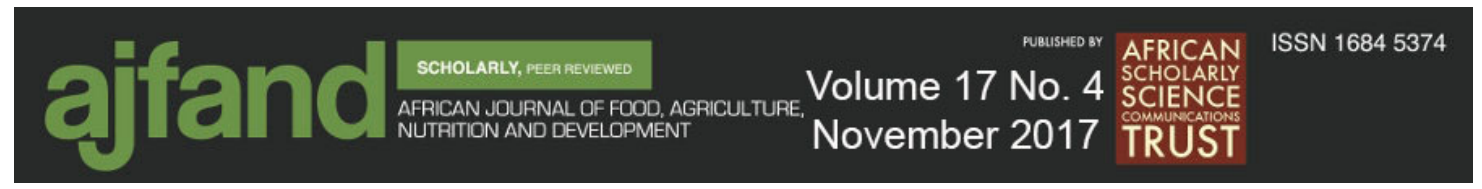

Age was determined from the child's date of birth as listed on their Under-5 Card. In instances where the caregiver did not have an Under-5 Card, the date of birth recorded in other documentation was used.

\section{Biochemical assessment}

Haemoglobin concentrations were measured with a portable photometer, the $\mathrm{HemoCue}^{\mathrm{TM}} 201+$, and a drop of venous blood as per manufacturer instructions [18]. Anaemia was defined as haemoglobin $(\mathrm{Hb})<110 \mathrm{~g} / \mathrm{L}$, with three levels of severity: mild anaemia corresponding to $\mathrm{Hb} 100-109 \mathrm{~g} / \mathrm{L}$, moderate anaemia corresponding to $\mathrm{Hb} 70-99 \mathrm{~g} / \mathrm{L}$, and severe anaemia corresponding to $\mathrm{Hb}<70 \mathrm{~g} / \mathrm{L}$ [14].

A slide was prepared with a thick blood smear of venous blood to diagnose malaria parasitism [19]. Presence of malaria parasites was determined by use of microscopy, analyzed by technicians at the Tropical Disease Research Centre (TDRC) in Ndola, Zambia [19]. Serum transferrin receptor (STfR) concentrations were analyzed using a sandwich enzyme-linked immunosorbent assay (ELISA) at the Erhardt Laboratory in Germany, with iron deficiency defined as STfR $>8.3 \mathrm{mg} / \mathrm{L}[20,21]$. Serum transferrin receptor is currently recommended by the WHO as the preferred indicator of iron deficiency in areas with a high disease burden, as it is less influenced by inflammation than serum ferritin [22]. Iron deficiency anaemia (IDA) was defined as concurrent iron deficiency and anaemia.

\section{Data analysis and statistical analysis}

After data collection, the data were entered into Excel spreadsheets. All of the data were then double checked by individuals different from those who originally entered it. The data were then transferred into IBM SPSS version 22.0.0.0 for analysis, with anthropometric indices calculated using WHO Anthro version 3.2.2. Descriptive tests consisted of frequencies for categorical variables and means and standard deviations for continuous variables.

A p-value $<0.05$ was used to determine significance for all tests. Independent samples t-tests were used to compare mean haemoglobin concentrations, and anthropometric z-scores between sub-groups including boys and girls, as well as subgroups determined from household characteristics and infant characteristics, such as presence or absence of fever. A Levene's test was conducted before performing t-tests to test the assumption of equality of variances between sub-groups.

Pearson's chi-square tests with a continuity correction, was used to compare the prevalence of anaemia, iron deficiency, stunting, wasting, and underweight between sub-groups. Odds ratios were used to compare the odds of having anaemia between different sub-groups, such as between boys and girls and between those who did or did not have fever. 


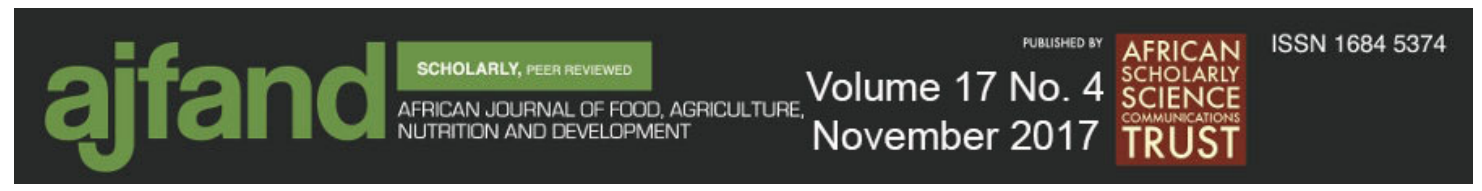

\section{RESULTS}

\section{Household and participant characteristics}

Table 1 shows household and participant characteristics. Approximately half of the infants, 318 of the total 631, were female, and the mean age was 9 (SD 2) months. The average age of the primary caregiver was 27 (SD 8) years. Nearly four fifths (78\%) of caregivers had achieved some level of formal education, with the majority having completed primary schooling only. Nearly all (91\%) households reported that they had some source of income, 97\% had land for cultivating crops, 53\% had a fruit and/or vegetable garden, and $82 \%$ owned a farm animal of some kind. Just over one third (36\%) of caregivers reported that they treated their water.

\section{IYCF knowledge and practices}

Infant and young child feeding knowledge and practices are reported in Table 2. Key findings were that every child had been breastfed at some point, and that breastfeeding had only been stopped for one child. Furthermore, the average age for the commencement of complementary feeding was six months, and it had commenced for $97 \%$ of the infants. It was found that $22 \%, 56 \%$ and $18 \%$ of infants were meeting minimum dietary diversity, minimum meal frequency, and minimum acceptable diet, respectively. Furthermore, 28\% had consumed an iron-rich food in the previous 24 hours.

\section{Morbidity and health characteristics of participants}

Table 3 shows morbidity and health characteristics of the infants. Caregivers reported that $56 \%$ of the infants had diarrhoea, $72 \%$ had a cough, and $56 \%$ had a fever over the previous two weeks. Over two thirds $(69 \%)$ of infants were sleeping under a mosquito net, and $10 \%$ tested positive for malaria parasites. Just under three quarters (74\%) had received a vitamin A capsule within the past six months.

\section{Anthropometrics, anaemia status and iron deficiency}

Table 4 presents results for stunting, wasting, underweight and MUAC of the infants recruited into the study. Overall, $30 \%$ were stunted, $2 \%$ were wasted, and $16 \%$ were underweight. There were no instances of severe wasting in the sample due to the exclusion criteria. Furthermore, $8 \%$ of the infants were severely stunted and $2 \%$ were severely underweight. Based on MUAC, $9 \%$ of the infants were suffering from moderate acute malnutrition. Due to the exclusion criteria there were no infants with MUAC $<11.5 \mathrm{~cm}$ or with bilateral oedema. Boys had higher rates of stunting $(\mathrm{p}=$ $0.003)$, severe stunting $(\mathrm{p}=0.005)$, and underweight than girls $(\mathrm{p}=0.001)$, although they had higher average MUAC values $(p<0.001)$. There was no significant difference in the prevalence of wasting according to sex.

Fifty-seven percent of the infants were anaemic, while $25 \%$ and $33 \%$ had mild and moderate anaemia, respectively (Table 5). There were no cases of severe anaemia as a result of the exclusion criteria for the study. Boys were more likely to be anaemic than girls $(\mathrm{p}=0.025)$. The overall mean $\mathrm{Hb}$ concentration was $105 \mathrm{~g} / \mathrm{L}(\mathrm{SD} 15)$. The mean $\mathrm{Hb}$ concentration was $103 \mathrm{~g} / \mathrm{L}$ (SD 15) for boys and 107 (SD 14) $\mathrm{g} / \mathrm{L}$ for girls $(\mathrm{p}=$ 0.001). Based on STfR concentrations, 93\% of infants were iron deficient, and $53 \%$ 


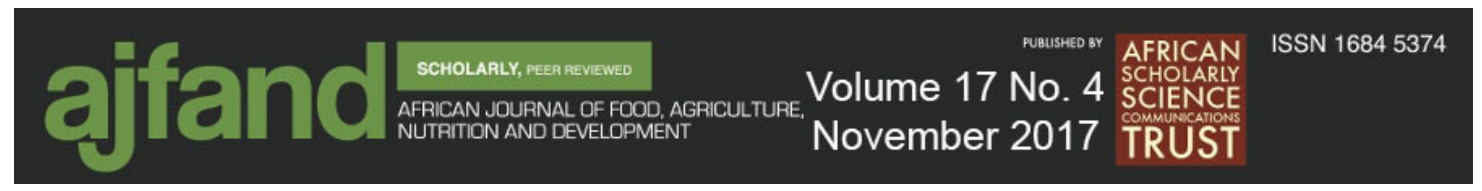

had IDA. Finally, as seen in Table 5, boys had a significantly higher prevalence of iron deficiency $(p=0.002)$ and of IDA $(p=0.002)$ than girls, as determined by Pearson's Chi-square test.

\section{Relationships between anaemia status and other variables}

Table 6 presents bivariate analysis comparing mean values of different continuous variables by anaemia status. Anaemic individuals had statistically significant ( $p<$ 0.05) lower ages and MUAC values than non-anaemic individuals. They also had higher average counts of malaria parasites in their blood per 200 white blood cells than non-anaemic individuals.

Table 7 presents the odds ratios for anaemia against various categorical characteristics of children in the sample. Being female, having received a vitamin A capsule in the previous 6 months, meeting minimum dietary diversity, the minimum acceptable diet, and having consumed iron-rich foods in the previous 24 hours were all associated with a significantly $(\mathrm{p}<0.05)$ lower risk of being anaemic, with the largest effect being achieving minimum dietary diversity, with an odds ratio of 0.44 (95\% CI: 0.30 0.65). Having had fever in the previous two weeks, having malaria parasitism, being underweight, or having moderate acute malnutrition as determined by MUAC were associated with statistically significant $(p<0.05)$ increases in the odds of being anaemic, with the largest effect being malaria parasitism, with an odds ratio of 4.33 (95\% CI: 2.16-8.70). There was no statistically significant difference in the odds of being anaemic whether or not the infant slept under a mosquito net.

As shown in Table 8 , there was a statistically significant $(p<0.05)$ relationship with haemoglobin concentration and sex as well as whether or not a household treated their water. Males had a lower average haemoglobin concentration as did households that did not treat their water. There was no statistically significant relationship between haemoglobin concentration and whether the caregiver had received formal education. Furthermore, there was a statistically significant $(p<0.05)$ relationship between haemoglobin concentration and whether or not the infant had diarrhoea or a fever in the previous two weeks. Infants who had diarrhoea in the previous two weeks had a $2.5 \mathrm{~g} / \mathrm{L}$ reduction in haemoglobin concentrations compared to those who did not have diarrhoea, and infants who had a fever in the previous two weeks had a $3.4 \mathrm{~g} / \mathrm{L}$ reduction in haemoglobin concentrations. There was no relationship between haemoglobin concentration and whether or not an infant slept under a mosquito net. Finally, infants with moderate acute malnutrition, as assessed by MUAC, had lower average haemoglobin concentrations.

\section{DISCUSSION}

\section{Prevalence of anaemia and iron deficiency}

Mild or moderate anaemia was found in $57 \%$ of the infants in this study, a major finding given their relatively young age. This is well above the $40 \%$ cut-off used by $\mathrm{WHO}$, to indicate a "severe" health crisis [2], although it is below the $70 \%$ rate of anaemia found nationally among infants under 12 months as reported in the 2012 Malaria Indicator Survey [3]. However, this higher prevalence found at the national 


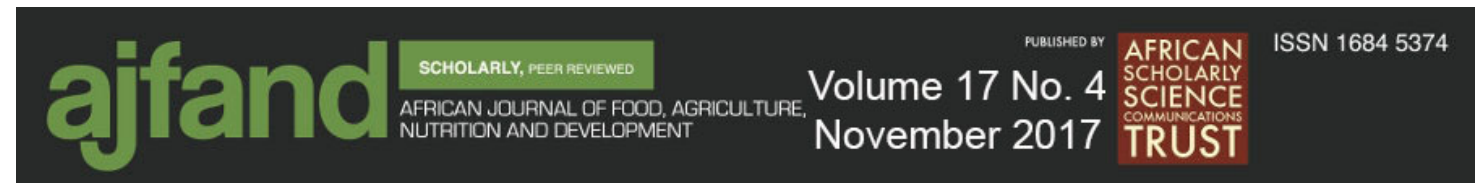

level also included severe anaemia, which was excluded from the study sample, making a direct comparison difficult.

There was a higher prevalence of moderate anaemia, which was found in $33 \%$ of the infants, as opposed to mild anaemia, which was found in $25 \%$ of the infants. The fact that moderate anaemia was found to be more prevalent than mild anaemia has implications for the health of the infants, as it indicates a higher level of morbidity. The $57 \%$ prevalence of anaemia reported here is most likely an underestimate of the true population prevalence, as the data does not include information on the prevalence of severe anaemia. A previous study in Zambia, using a nationally representative sample, found a prevalence of severe anaemia of only $2 \%$, but as this study is from 2003, its usefulness is limited in estimating the effect that exclusion criteria had on the prevalence of anaemia found in this sample [6].

There was a very high prevalence of iron deficiency (93\%) based on concentrations of $\mathrm{STfR}>8.3 \mathrm{mg} / \mathrm{L}$. This is much higher than has been reported in other Zambian studies, although as they were using different methods of assessment and were in different regions, it is difficult to make a direct comparison $[7,9,10,11]$. Furthermore, there is debate in the literature as to the proper cut- off to use for STfR, and thus complicating interpretation [22].

\section{Household and participant characteristics}

Based on caregiver reports there was a high burden of disease in the population. In the previous two weeks, $56 \%$ of the infants had diarrhoea, $72 \%$ had cough, and $56 \%$ had fever. This is in contrast to findings from both the Zambian 2013-2014 Demographic Health Survey (DHS), which found a $26 \%$ prevalence of fever for children under 5 years in the Northern Province and a $25 \%$ prevalence nationally among children 6-11 months [23]. The DHS also reported a $16 \%$ prevalence rate for diarrhoea among children under 5 years in the Northern Province and a $29 \%$ prevalence nationally among infants 6-11 months [23].

The $10 \%$ malaria parasitism burden in the population matches the findings from the 2012 Zambian Malaria Indicator Survey, which found a 10\% rate of parasitism nationally in infants under 12 months of age [3]. The proportion of infants under 12 months sleeping under a mosquito net the previous night, at $69 \%$, was roughly the same in both the present study and nationally as reported in the Malaria Indicator Survey [3]. It should be noted that as this study took place during the dry season: malaria parasitism was likely lower than during other times of the year, as there is a seasonal pattern to malaria transmission in Zambia [3].

The dietary quality of the infants in the sample was better than that seen nationally, based on the proportions of infants achieving the minimum dietary diversity and the minimum acceptable diet, which are $22 \%$ and $11 \%$ nationally, and $22 \%$ and $18 \%$ in the study sample [23]. Likewise, the proportion of infants achieving the minimum meal frequency was higher, at $56 \%$, than the national rate of $42 \%$ [23]. Nationally, $24 \%$ of infants 6-8 months, and $49 \%$ of infants 9-11 months consumed iron rich foods the previous day, compared to $28 \%$ of infants in the sample [23]. However, as the 


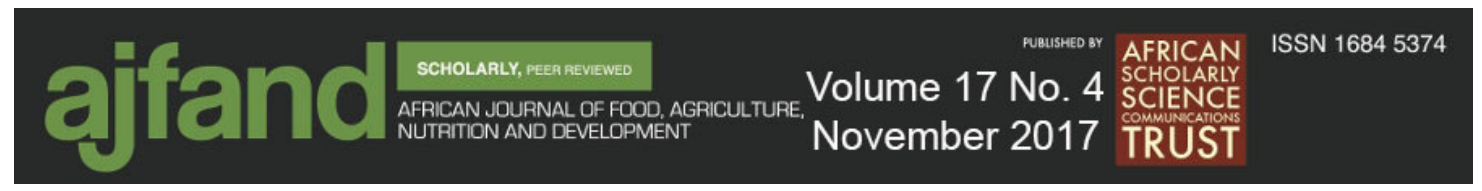

information in this study is based on a single time point, it is possible that there are seasonal patterns to dietary quality not apparent in the data.

The prevalence of stunting, at 30\%, was similar to findings at the national level, in which $25 \%$ of infants 6-8 months and 39\% of infants 9-11 months were stunted, although the study sample does seem to have a lower prevalence of severe stunting [23]. The finding that boys are more stunted than girls has been found nationally [23]. In fact, this relationship has been found to be statistically significant across much of English-speaking sub-Saharan Africa, although the reasons for this are unknown [24]. There was a lower prevalence of moderate wasting in the sample, at $2 \%$, than found nationally, where it was found that 7\% of infants $6-8$ months and $8 \%$ of infants $9-11$ months were moderately wasted [23]. Due to the exclusion of individuals with severe wasting from the study, it was not possible to comment on how it may compare to national findings. The study sample was similar in terms of underweight when compared nationally. While $16 \%$ of infants in the study were underweight, only $11 \%$ of infants 6-8 months and 17\% of children 9-11 months were underweight nationally in the previous DHS [23].

\section{Factors associated with anaemia status and haemoglobin concentrations}

Both the age of infants and their sex had an effect on both haemoglobin concentrations, and anaemia status. A relationship between age, haemoglobin concentration, and anaemia status is in line with the 2012 Zambian Malaria Indicator Survey, which found a decreasing prevalence of anaemia as age increased [3]. This has also been seen in Zanzibar and Nepal $[25,26]$. There were higher concentrations of haemoglobin in girls, who had an odds ratio for anaemia of 0.69 when compared to boys. A relationship between anaemia and sex was also found in Timor-Leste [27].

There was no relationship between the educational status of the primary caregiver and the infants' haemoglobin concentration or anaemia status. While this has previously been found in Zambia [6], it is distinct from results from Tanzania, in which a relationship was found [28]. Furthermore, there was no relationship between whether or not the primary caregiver had previously heard of anaemia and the risk of their infant being anaemic; this is in contrast to a study from Indonesia [29].

In households that treated their water to make it safer to drink, infants had a mean haemoglobin concentration that was $3 \mathrm{~g} / \mathrm{L}$ higher than those living in households that did not treat their water $(p=0.021)$; however, the overall effect of water treatment on whether or not an infant was anaemic was not significant. The effect of water treatment on haemoglobin status has been reported elsewhere [30]. This may be related to the effect of infection and illness on haemoglobin status [31], specifically the potential association between water quality and diarrhoea [32]. Notably, diarrhoea in the previous two weeks was associated with decreased haemoglobin concentrations, paralleling results in Timor-Leste and Brazil [27, 30].

Fever was associated with lower concentrations of haemoglobin and a higher risk of anaemia, with one possible explanation being the effect of malaria. Malaria parasitism was associated with a greatly increased risk of being anaemic $(\mathrm{OR}=4.33)$. This 


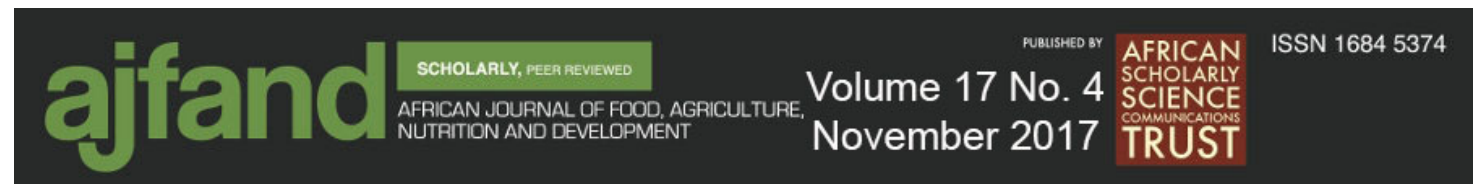

parallels other Zambian studies which found an association between malaria and anaemia status [5-8]. Despite this, there was no association between haemoglobin concentrations or anaemia status and whether or not an infant had slept under a mosquito net. While counterintuitive, this was also a result in a study from the Luangwa District of Zambia [8].

Infants with MUAC above $12.5 \mathrm{~cm}$ had an average haemoglobin concentration of 106 $\mathrm{g} / \mathrm{L}$ whereas infants with MUAC between $11.5 \mathrm{~cm}$ and $12.5 \mathrm{~cm}$, indicative of moderate acute malnutrition, had average haemoglobin concentrations of only $100 \mathrm{~g} / \mathrm{L}$. The risk of being anaemic was 2.5 times greater for infants with moderate acute malnutrition than those without. Previous research has found an association between underweight and anaemia in South African infants 6-11 months [33].

Infants achieving minimum dietary diversity had a lower risk of being anaemic than those who were not, as was the case in research from China [34]. This likely reflects the impact of micronutrient deficiencies on haemoglobin concentrations as dietary diversity has been found to be a predictor of the micronutrient density and a number of micronutrient deficiencies have been linked to anaemia [35, 36]. Furthermore, infants who had consumed iron-rich foods in the previous 24 hours were less likely to be anaemic $(\mathrm{OR}=0.56)$, pointing to the importance of iron in the pediatric diet. While individuals who were iron-deficient were not more likely to be anaemic than those who were not iron-deficient, anaemic individuals did have higher concentrations of STfR, as expected.

\section{CONCLUSION}

There were limitations to study design which may have affected the results: the lack of randomization, and the exclusion of infants with severe anaemia, which make it harder to draw broad population-wide conclusions, and the fact that certain covariates, such as haemoglobinopathies, and infections with helminths were not measured. However, the findings suggest the importance of diet, malaria, and infection in understanding the etiology of anaemia in this region of Zambia. Less clear, but still worthy of further research, is the potential relationship between anaemia and sanitation suggested by the findings related to water treatment and diarrhoea. This information is critical as there is currently only limited research on the specific etiology of anaemia in the Zambian setting, particularly for this age group. It can be used to implement future interventions as well as to guide the design of studies intended to establish causal pathways.

Overall, the high prevalence of anaemia and iron deficiency found in the sample highlights the importance of continued efforts to understand their specific etiology in the Zambian context as well as the importance of programs aimed at tackling them. Recommended interventions could focus on sanitation and water quality, improving the quality of the diet through diversification and fortification and reducing the prevalence of diseases such as malaria. Future studies should examine haemoglobinopathy, and infections with helminthes to gain a better sense of their impact in Zambia. 


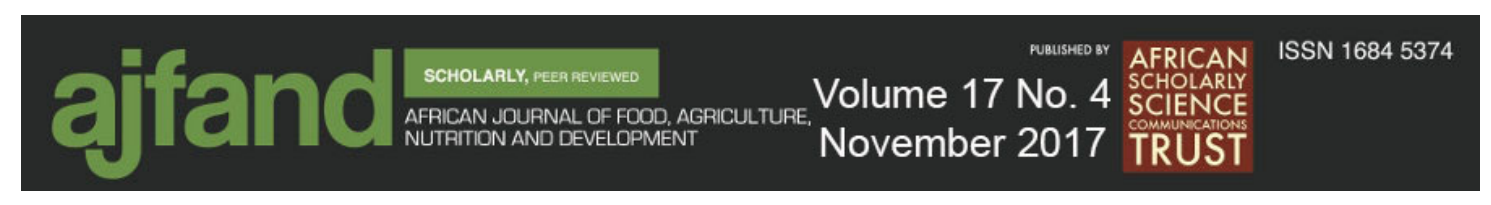

\section{ACKNOWLEDGEMENTS}

We are thankful for the funding from Irish Aid and UNICEF. We thank Ruth Siyandi at UNICEF-Zambia for her assistance throughout. We are also thankful for the assistance from the staff of the Provincial Medical Office, and the District Medical Office in Zambia as well as the staff at the National Food and Nutrition Commission for sensitization and community mobilization. We also thank Justin Chileshe and staff at the Tropical Research Disease Center in Ndola, Zambia, and Dr. Juergen Erhardt from the Erhardt Laboratory in Germany for the analysis of blood samples. We also thank Diane Kim, and the enumerators for assisting in data collection. 


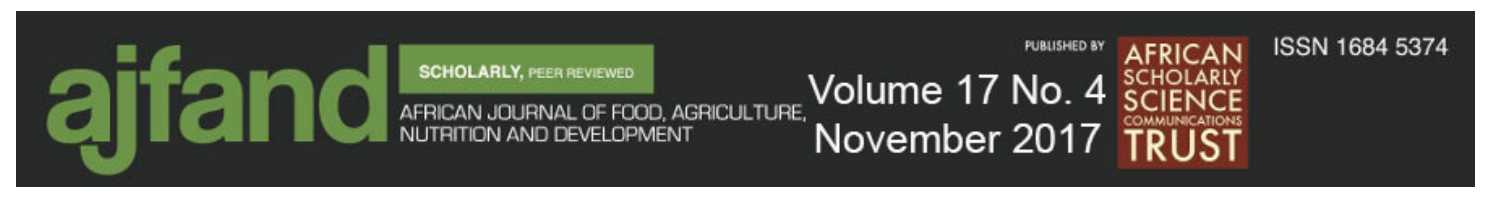

Table 1: Household and participant characteristics

\begin{tabular}{lcc}
\hline \multicolumn{1}{c}{ Characteristic } & Value & Total N \\
\hline Female, \% (n) & $50.3 \%(318)$ & 631 \\
Age of infant, months, mean (SD) & $8.9(1.7)$ & 631 \\
Age of primary caregiver in years, mean (SD) & $26.7(7.6)$ & 631 \\
Household size, mean (SD) & $5.9(2.4)$ & 630 \\
Number of children under 5 in household, mean (SD) & $3.3(2.2)$ & 614 \\
Education level of primary caregiver & & \\
$\bullet \quad$ No school, \% (n) & $21.7 \%(137)$ & 631 \\
- Primary (Grade 1-7), \% (n) & $59.3 \%(374)$ & 631 \\
- Secondary (Grade 8-12), \% (n) & $17.7 \%(112)$ & 631 \\
- Tertiary (University or higher), \% (n) & $0.5 \%(3)$ & 631 \\
Education level not specified, \% (n) & $0.8 \%(5)$ & 631 \\
Household has source of income, \% (n) & & \\
Household has land for cultivating crops, \% (n) & $90.6 \%(569)$ & 628 \\
Household has fruit and/or vegetable garden, \% (n) & $96.5 \%(609)$ & 631 \\
Household has livestock/farm animals, \% (n) & $52.6 \%(332)$ & 630 \\
Household treats water to make it safer, \% (n) & $81.6 \%(511)$ & 626 \\
Household boils water, \% (n) & & \\
Household treats water with bleach/chlorine, \% (n) & $26.6 \%(227)$ & 631 \\
Household treats water, method not specified, \%(n) & $0.6 \%(4)$ & 631 \\
Adults wash hands with soap or ash, \% (n) & $86.5 \%(545)$ & 630 \\
\hline
\end{tabular}




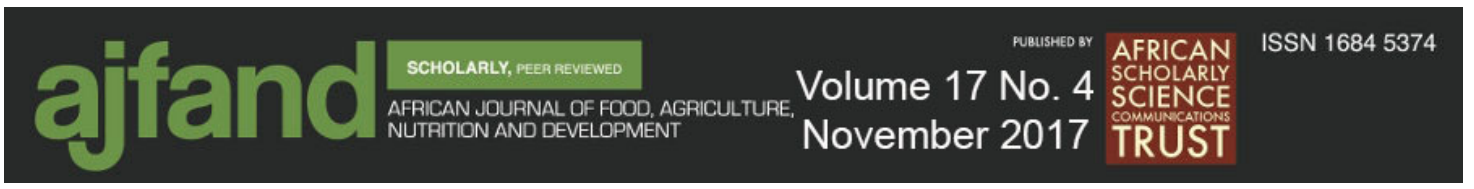

Table 2: Infant and young child feeding (IYCF) knowledge and practices of caregivers in Mbala District

\begin{tabular}{|c|c|c|}
\hline IYCF knowledge/practice & Value & Total N \\
\hline Infant currently breastfed, \% (n) & $99.8 \%(630)$ & 631 \\
\hline Infant ever breastfed, $\%$ (n) & $100 \%(631)$ & 631 \\
\hline Infant has commenced complementary feeding, \% (n) & $96.5 \%(609)$ & 631 \\
\hline Age of start of complementary feeding, months, mean (SD) & $5.9(1.1)$ & 609 \\
\hline Infant meeting minimum dietary diversity ${ }^{1}, \%(n)$ & $22.0 \%(139)$ & 631 \\
\hline Infant meeting minimum meal frequency ${ }^{2}, \%(n)$ & $56.1 \%(352)$ & 628 \\
\hline Infant meeting minimum acceptable $\operatorname{diet}^{3}, \%(n)$ & $18.0 \%(113)$ & 628 \\
\hline Infant consuming iron-rich food in previous 24 hours ${ }^{4}, \%$ (n) & $28.2(178)$ & 631 \\
\hline Caregiver heard of anaemia and/or iron deficiency, \% (n) & $87.0(549)$ & 631 \\
\hline Caregiver spoken to by healthcare workers about IYCF, \% (n) & $69.9 \%(441)$ & 631 \\
\hline
\end{tabular}

${ }^{1}$ Defined as consuming at least 4 of grains, roots and tubers; legumes and nuts; dairy products; flesh foods; eggs; vitamin A rich fruits and vegetables; or other fruits and vegetables

${ }^{2}$ Defined as 2 meals or snacks for breastfed infants 6-8 months, 3 meals or snacks for breastfed children 9-23 months, and 4 meals or snacks for non-breastfed children 6-23 months

${ }^{3}$ Defined as at least 2 milk feedings and the minimum dietary diversity not including milk feeds and the minimum meal frequency for non-breastfed children 6-23 months and as minimum dietary diversity and minimum meal frequency for breastfed infants 6-23 months

${ }^{4}$ Defined as flesh foods, commercially fortified foods specially designed for infants and young children that contain iron

Table 3: Morbidity and health of infants 6-11 months in Mbala District

\begin{tabular}{lcc}
\hline \multicolumn{1}{c}{ Morbidity/health Characteristic } & Value & Total N \\
\hline Diarrhoea in the past 2 weeks ${ }^{1}, \%(\mathrm{n})$ & $56.1 \%(354)$ & 631 \\
Cough in the past 2 weeks, \% (n) & $72.1 \%(455)$ & 631 \\
Fever in the past 2 weeks, \% (n) & $55.6 \%(349)$ & 628 \\
More than one of the above in the past 2 weeks, \% (n) & $63.9 \%(401)$ & 628 \\
None of the above in the past 2 weeks, \% (n) & $12.6 \%(79)$ & 628 \\
& & \\
Infants sleeping under a mosquito net, \% (n) & $68.9 \%(433)$ & 628 \\
Infants with fever who were tested for malaria, \% (n) & $46.6 \%(129)$ & 277 \\
Infants who are currently being treated for malaria, \% (n) & $9.2 \%(58)$ & 631 \\
Infants with malaria parasitism, \% (n) & $9.9 \%(62)$ & 625 \\
Malaria parasites per 200 white blood cells, mean (range) & $23.6(0-4000)$ & 625 \\
& & \\
Given a vitamin A capsule within past 6 months, \% (n) & $73.7 \%(465)$ & 631 \\
Given a de-worming capsule within past 6 months, \% (n) & $10.3 \%(65)$ & 631 \\
\hline Defined as 3 or more times a day of loose stools & &
\end{tabular}




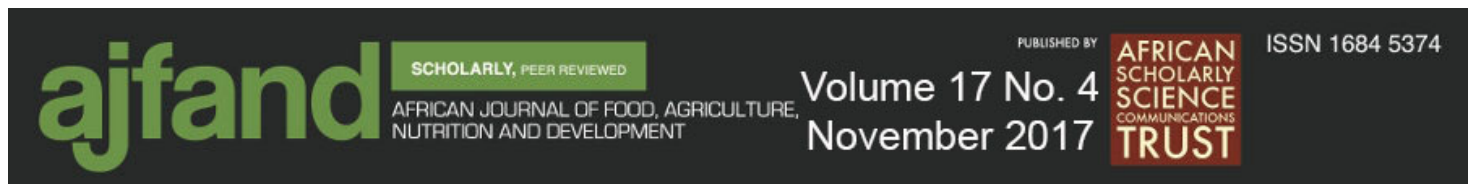

Table 4: Stunting, wasting, underweight and mid-upper arm circumference (MUAC) of male and female infants 6-11 months in Mbala District

\begin{tabular}{|c|c|c|c|c|}
\hline Anthropometric characteristic & Male & Female & p-value ${ }^{1}$ & Overall \\
\hline Stunted (height-for-age z-score $<-2$ ), $\%$ (n) & $35.6 \%(111)$ & $24.5 \%(78)$ & $0.003 *$ & $30.0 \%(189)$ \\
\hline Severely stunted (height-for-age z-score $<-3$ ), \% (n) & $10.6 \%(33)$ & $4.4 \%(14)$ & $0.005^{*}$ & $7.5 \%(47)$ \\
\hline Height-for-age z-score, mean (SD) & $-1.7(1.1)$ & $-1.4(1.0)$ & $0.001 *$ & $-1.5(1.1)$ \\
\hline Wasted (weight-for-height z-score $<-2$ ), $\%$ (n) & $2.6 \%(8)$ & $2.2 \%(7)$ & 0.980 & $2.4 \%(15)$ \\
\hline Weight-for-height z-score, mean (SD) & $-0.1(1.1)$ & $0.0(1.0)$ & 0.203 & $0.0(1.0)$ \\
\hline Underweight (weight-for-age z-score $<-2$ ), \% (n) & $21.1 \%(66)$ & $10.8 \%(34)$ & $0.001 *$ & $15.9 \%(100)$ \\
\hline Severely underweight (weight-for-age z-score $<-3$ ), \% (n) & $2.2 \%(7)$ & $1.6 \%(5)$ & 0.758 & $1.9 \%(12)$ \\
\hline Weight-for-age z-score, mean (SD) & $-1.0(1.1)$ & $-0.8(1.0)$ & $0.005^{*}$ & $-0.9(1.1)$ \\
\hline Moderate acute malnutrition (MUAC $11.5-12.5 \mathrm{~cm}$ ), \% (n) & $6.7 \%(21)$ & $11.3(36)$ & 0.060 & $9.0 \%(57)$ \\
\hline MUAC (Mid upper-arm circumference), mean (SD) & $13.9(1.0)$ & $13.6(1.0)$ & $<0.001^{*}$ & $13.8(1.0)$ \\
\hline
\end{tabular}

1 Comparing male and female infants, Chi-square test used for categorical variables and independent sample t-test for continuous

* Significant at $\mathrm{p}<0.05$ 


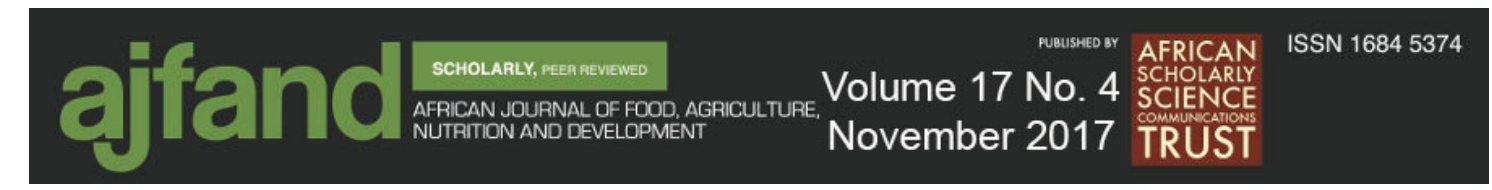

Table 5: Haemoglobin (Hb) concentrations, anaemia status, and iron deficiency as assessed by serum transferrin receptor (STfR) of infants 6-11 months in Mbala District

\begin{tabular}{|c|c|c|c|c|}
\hline Characteristic & $\begin{array}{c}\text { Male } \\
(\mathrm{N}=313)\end{array}$ & $\begin{array}{c}\text { Female } \\
(\mathrm{N}=318)\end{array}$ & p-value ${ }^{1}$ & Overall \\
\hline Anaemic $(\mathrm{Hb}<110 \mathrm{~g} / \mathrm{L}), \%(\mathrm{n})$ & $62.0 \%(194)$ & $52.8 \%(168)$ & $0.025^{*}$ & $57.4 \%(362)$ \\
\hline Mildly anaemic (Hb 100-109 g/L), \% (n) & $24.0 \%(75)$ & $25.5 \%(81)$ & 0.728 & $24.7 \%(156)$ \\
\hline Moderately anaemic $(\mathrm{Hb} 70-99 \mathrm{~g} / \mathrm{L}), \%$ (n) & $38.0 \%(119)$ & $27.4 \%(87)$ & $0.006^{*}$ & $32.6 \%(206)$ \\
\hline $\mathrm{Hb}$ concentration $(\mathrm{g} / \mathrm{L})$, mean $(\mathrm{SD})$ & $103.2(15.3)$ & $107.1(14.0)$ & $0.001 *$ & $105.2(15.2)$ \\
\hline Iron deficient $(\mathrm{STfR}>8.3 \mathrm{mg} / \mathrm{L}), \%$ (n) & $96.1 \%(295)$ & $89.5 \%(280)$ & $0.002 *$ & $92.7 \%(575)$ \\
\hline Iron deficiency anaemia (IDA), \% (n) & $59.3 \%(182)$ & $47.0 \%(147)$ & $0.002 *$ & $53.1 \%(329)$ \\
\hline
\end{tabular}

1 Comparing male and female infants, Chi-square test used for categorical variables and independent sample t-test for continuous

* Significant at $\mathrm{p}<0.05$ 


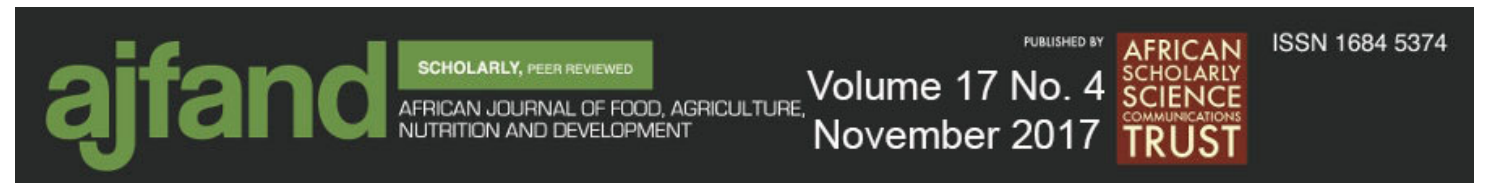

Table 6: Comparison of anaemia status against continuous participant, household, morbidity, and anthropometric and characteristics in a sample of 6-11 month old infants in Mbala District

\begin{tabular}{lccccc}
\hline & \multicolumn{3}{c}{ Anaemic } & \multicolumn{2}{c}{ Not anaemic } \\
\multicolumn{1}{c}{ Characteristic } & Mean & SD & Mean & SD & p-value \\
\hline Age (months) & 8.7 & 1.8 & 9.1 & 1.6 & $0.001^{2 *}$ \\
Age of caregiver (years) & 26.3 & 7.1 & 27.2 & 8.3 & 0.162 \\
Number of individuals in household & 5.9 & 2.4 & 5.9 & 2.3 & 0.963 \\
Number of children under 5 in household & 3.4 & 2.3 & 3.3 & 2.1 & 0.571 \\
Malaria parasites per 200 white blood cells & 37.7 & 287.6 & 4.6 & 70 & $0.037^{*}$ \\
Start of complementary feeding (months) & 5.8 & 1.1 & 5.9 & 1.0 & 0.137 \\
Serum transferrin receptor (mg/L) & 17.6 & 8.1 & 13.4 & 4.3 & $<0.001^{2 *}$ \\
Wasting (weight-for-height z-score) & -0.1 & 1.1 & 0 & 1.0 & 0.625 \\
Stunting (height-for-age z-score) & -1.6 & 1.1 & -1.4 & 1.0 & 0.115 \\
Underweight (weight-for-age z-score) & -1.0 & 1.1 & -0.9 & 1.0 & 0.092 \\
Mid-upper arm circumference (cm) & 13.7 & 1.1 & 13.9 & 1.0 & $0.036^{*}$ \\
\hline 1 Calculated from comparing means using an independent samples t-test & & \\
2 Equal variables between groups not assumed & & & & & \\
* Significant at $\mathrm{p}<0.05$ & & & & &
\end{tabular}




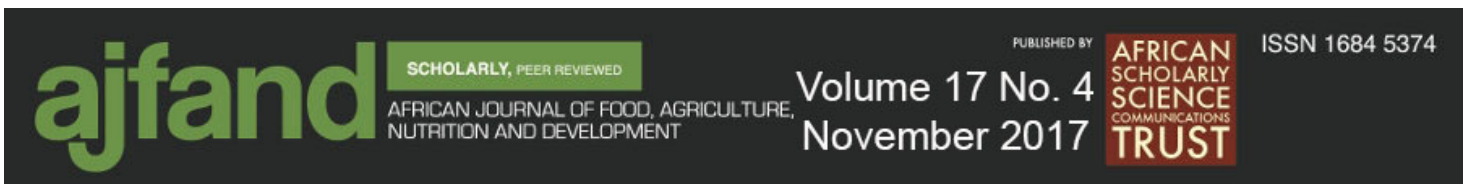

Table 7: Comparison of anaemia status against categorical participant, household, morbidity, and anthropometric characteristics in a sample of 6-11 month old infants in Mbala District

\begin{tabular}{|c|c|c|}
\hline Characteristic & $\begin{array}{c}\text { Odds Ratio } \\
(95 \% \text { CI })\end{array}$ & Total N \\
\hline Sex (female versus male) & $0.69(0.50-0.94)^{*}$ & 631 \\
\hline Caregiver has attended school & $0.95(0.65-1.39)$ & 631 \\
\hline Household has source of income & $0.61(0.35-1.08)$ & 628 \\
\hline Household has land for cultivation & $1.13(0.48-2.65)$ & 631 \\
\hline Household has garden for fruit and/or vegetables & $0.81(0.59-1.11)$ & 630 \\
\hline Household owns animals or livestock & $1.09(0.72-1.64)$ & 626 \\
\hline Household treats water & $0.86(0.62-1.2)$ & 631 \\
\hline Caregiver washes hands with soap or ash & $0.88(0.55-1.4)$ & 630 \\
\hline Infant diarrhoea in the previous two weeks & $1.26(0.92-1.74)$ & 631 \\
\hline Infant cough in the previous two weeks & $1.10(0.78-1.56)$ & 631 \\
\hline Infant fever in the previous two weeks & $1.88(1.36-2.59)^{*}$ & 628 \\
\hline Infant sleeps under a mosquito net & $0.79(0.56-1.11)$ & 628 \\
\hline Infant has malaria parasites & $4.33(2.16-8.70)^{*}$ & 625 \\
\hline Received a vitamin A capsule in previous 6 months & $0.68(0.47-0.99)^{*}$ & 628 \\
\hline Received a deworming tablet in previous 6 months & $0.85(0.51-1.42)$ & 629 \\
\hline Infant has begun complementary feeding & $0.62(0.25-1.54)$ & 631 \\
\hline Infant meeting minimum dietary diversity & $0.44(0.30-0.65)^{*}$ & 631 \\
\hline Infant meeting minimum meal frequency & $0.92(0.67-1.26)$ & 628 \\
\hline Infant meeting minimum acceptable diet & $0.57(0.38-0.86)^{*}$ & 628 \\
\hline Infant has consumed iron rich food previous 24 hours & $0.56(0.40-0.80)^{*}$ & 631 \\
\hline Caregiver has heard of anaemia & $0.95(0.59-1.52)$ & 631 \\
\hline Caregiver has been spoken to about IYCF & $1.36(0.96-1.91)$ & 631 \\
\hline Iron deficient (serum transferrin receptor $>8.3 \mathrm{mg} / \mathrm{L}$ ) & $1.17(0.64-2.15)$ & 620 \\
\hline Stunted (height-for-age z-score $<-2$ ) & $1.35(0.96-1.92)$ & 630 \\
\hline Wasted (weight-for-height z-score $<-2$ ) & $1.50(0.51-4.45)$ & 628 \\
\hline Underweight (weight-for-age z-score < -2) & $1.71(1.09-2.70)^{*}$ & 629 \\
\hline Moderate acute malnutrition (MUAC $<12.5 \mathrm{~cm}$ ) & $2.46(1.31-4.59)^{*}$ & 631 \\
\hline
\end{tabular}

* Significant at $\mathrm{p}<0.05$ 


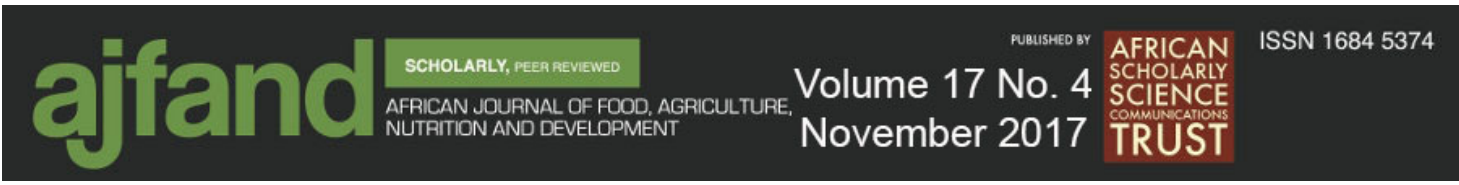

Table 8: Comparison of haemoglobin $(\mathrm{Hb})$ concentration against categorical household and participant characteristics in a sample of 6-11 month old infants in Mbala District

\begin{tabular}{|c|c|c|c|}
\hline Characteristic & Mean $\mathrm{Hb}(\mathrm{g} / \mathrm{L})$ & SD & $\mathrm{p}$-value $^{1}$ \\
\hline Male & 103.2 & 15.3 & \multirow{2}{*}{$0.001 *$} \\
\hline Female & 107.1 & 14.9 & \\
\hline Caregiver has attended school & 105.4 & 15.3 & \multirow{2}{*}{0.511} \\
\hline Caregiver has not attended school & 104.4 & 14.8 & \\
\hline Household has source of income & 105.5 & 15.3 & \multirow{2}{*}{0.136} \\
\hline Household has no source of income & 102.4 & 14.0 & \\
\hline Household has land for cultivation & 105.1 & 15.2 & \multirow{2}{*}{0.762} \\
\hline Household has no land for cultivation & 106.1 & 15.6 & \\
\hline Household has garden for fruit and/or vegetables & 105.7 & 15.9 & \multirow{2}{*}{0.365} \\
\hline Household has no garden for fruit and/or vegetables & 104.6 & 14.4 & \\
\hline Household owns animals or livestock & 105.3 & 15.4 & \multirow{2}{*}{0.634} \\
\hline Household owns no animals or livestock & 104.6 & 14.7 & \\
\hline Household treats water & 107.0 & 15.7 & \multirow{2}{*}{$0.021 *$} \\
\hline Household does not treat water & 104.1 & 14.8 & \\
\hline Infant diarrhoea in the previous two weeks & 104.1 & 15.6 & \multirow{2}{*}{$0.042 *$} \\
\hline Infant diarrhoea free in the previous two weeks & 106.6 & 14.5 & \\
\hline Infant had a cough in the previous two weeks & 104.9 & 15.3 & \multirow{2}{*}{0.421} \\
\hline Infant cough free in the previous two weeks & 106.0 & 14.9 & \\
\hline Infant fever in the previous two weeks & 103.6 & 15.3 & \multirow{2}{*}{$0.005^{*}$} \\
\hline Infant fever free in the previous two weeks & 107.0 & 14.9 & \\
\hline Infant sleeps under a mosquito net & 105.3 & 15 & \multirow{2}{*}{0.626} \\
\hline Infant does not sleep under a mosquito net & 104.7 & 15.6 & \\
\hline Stunted (height-for-age z-score $<-2$ ) & 103.4 & 15.1 & \multirow{2}{*}{0.055} \\
\hline Not Stunted (height-for-age $\mathrm{z}$-score $\geq-2$ ) & 106.0 & 15.2 & \\
\hline Wasted (weight-for-height z-score $<-2$ ) & 104.7 & 14.8 & \multirow{2}{*}{0.902} \\
\hline Not Wasted (weight-for-height $\mathrm{z}$-score $\geq-2$ ) & 105.2 & 15.2 & \\
\hline Underweight (weight-for-age z-score $<-2$ ) & 102.7 & 12.9 & \multirow{2}{*}{0.078} \\
\hline Not Underweight (weight-for-age z-score $\geq-2$ ) & 105.7 & 15.6 & \\
\hline No moderate acute malnutrition (MUAC $\geq 12.5 \mathrm{~cm}$ ) & 105.7 & 15.2 & \multirow{2}{*}{$0.004^{*}$} \\
\hline Moderate acute malnutrition (MUAC $<12.5 \mathrm{~cm}$ ) & 99.7 & 14.5 & \\
\hline
\end{tabular}




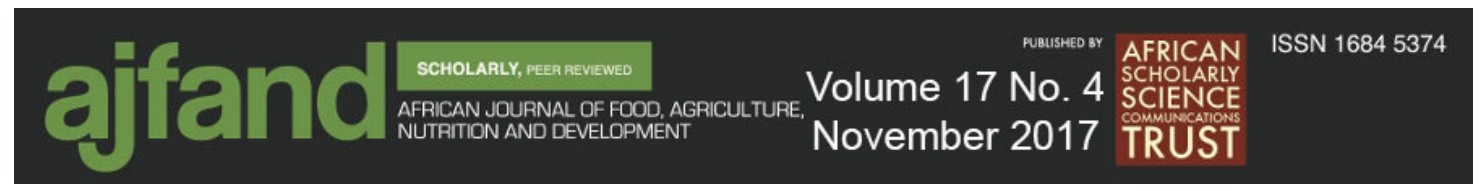

\section{REFERENCES}

1. Dewey $\mathbf{K}$ and $\mathbf{K}$ Begum Long term consequences of stunting in early life. Matern Child Nutr. 2011; 7: 5-18.

2. WHO. Worldwide prevalence of anaemia 1993-2005. World Health Organization, Geneva, Switzerland; 2008.

3. Ministry of Health. Zambia National Malaria Indicator Survey 2012. Lusaka, Zambia; 2013.

4. Clark S Iron deficiency anemia. Nutr Clin Pract. 2008; 23(2): 128-41.

5. Luo C, Mwela $\mathbf{C}$ and $\mathbf{J}$ Campbell National baseline survey on prevalence and aetiology of anaemia in Zambia: a random cluster community survey involving children, women and men. Lusaka, Zambia; 1999.

6. Clewes $\mathbf{C}$ and $\mathbf{C}$ Kankasa Report of the National Survey to Evaluate the Impact of Vitamin A Interventions in Zambia In July and November 2003. 2005.

7. Van Dijk J, Hachaambwa L, Mulenga M, Mharakurwa $\mathbf{S}$ and $\mathbf{P}$ Thuma Response of Haemoglobin Concentration to Oral Supplemental Iron in Children living in a Malaria endemic area of Zambia. Med J Zambia. 2007; 34(2): 86-91.

8. Eisele TP, Miller JM, Moonga HB, Hamainza $\mathbf{B}$, Hutchinson $\mathbf{P}$ and $\mathbf{J}$ Keating Malaria infection and anemia prevalence in Zambia's Luangwa District: an area of near-universal insecticide-treated mosquito net coverage. Am J Trop Med Hyg. 2011; 84(1): 152-7.

9. Gibson RS, Kafwembe E, Mwanza S, Gosset L, Bailey KB, Mullen A, Baisley $\mathbf{L}$ and $\mathbf{S}$ Filteau A Micronutrient-Fortified Food Enhances Iron and Selenium Status of Zambian Infants but Has Limited Efficacy on Zinc. J Nutr. 2011; 141: 935-43.

10. Van Rheenen PF, de Moor LTT, Eschbach S and BJ Brabin A cohort study of haemoglobin and zinc protoporphyrin levels in term Zambian infants: effects of iron stores at birth, complementary food and placental malaria. Eur $J$ Clin Nutr. 2008; 62(12): 1379-87.

11. Bresnahan K, Chileshe J, Arscott S, Nuss E, Surles R, Masi C, Kafwembe E and AT Sherry The Acute Phase Response Affected Traditional Measures of Micronutrient Status in Rural Zambian Children during a Randomized, Controlled Feeding Trial. J Nutr. 2014; 144: 972-8. 


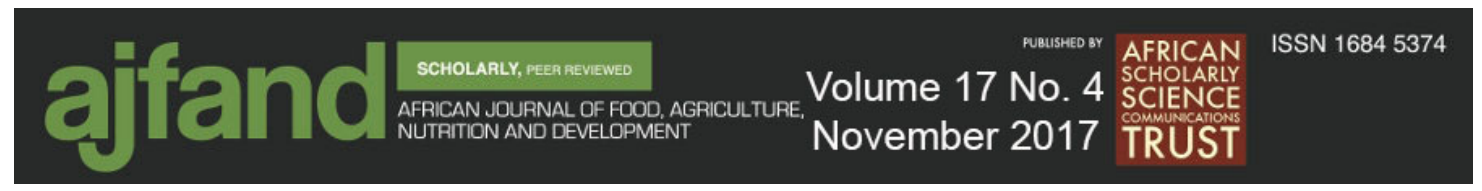

12. Van Dongen PWJ and MA van't Hof Sickle cell trait, malaria and anaemia in pregnant Zambian women. Trans R Soc Trop Med Hyg. 1983; 77(3): 402-4.

13. WHO and UNICEF. WHO child growth standards and the identification of severe acute malnutrition in infants and children. World Health Organization, Geneva, Switzerland; 2009.

14. WHO VMNIS. Haemoglobin concentrations for the diagnosis of anaemia and assessment of severity. World Health Organization, Geneva, Switzerland; 2011.

15. WHO. Indicators for assessing infant and young child feeding practices. Part 3: Country Profiles. World Health Organization, Geneva, Switzerland; 2010.

16. WHO Multicentre Growth Reference Study Group. WHO Child Growth Standards: Length/height-for-age, weight-for-age, weight-for-length, weightfor-height and body mass index-for-age: Methods and development. World Health Organization, Geneva, Switzerland. 2006.

17. HemoCue AB HemoCue $\mathrm{Hb}$ 201+ Operating Manual. HemoCue AB, Ängelholm, Sweden. N.D.

18. Nkrumah B, Nguah SB, Sarpong N, Dekker D, Idriss A, May J and Y Adu-Sarkodie Hemoglobin estimation by the HemoCue ${ }^{\circledR}$ portable hemoglobin photometer in a resource poor setting. BMC Clinical Pathology. 2011; 11(1): 5 .

19. WHO. Basic Malaria Microscopy: Part 1 Learner's Guide, Second Edition. World Health Organization, Geneva, Switzerland; 2010.

20. Erhardt JG, Estes JE, Pfeiffer CM, Biesalski HK and NE Craft Combined measurement of ferritin, soluble transferrin receptor, retinol binding protein, and C-reactive protein by an inexpensive, sensitive, and simple sandwich enzyme-linked immunosorbent assay technique. J Nutr. 2004; 134(11): $3127-$ 3132 .

21. Engle-Stone R, Nankap M, Ndjebayi A, Erhardt JG and K Brown Plasma Ferritin and Soluble Transferrin Receptor Concentrations and Body Iron Stores Identify Similar Risk Factors for Iron Deficiency but Result in Different Estimates. J Nutr. 2013; 143(8): 369-77.

22. WHO VMNIS. Serum transferrin receptor levels for the assessment of iron status and iron deficiency in populations. World Health Organization, Geneva, Switzerland; 2014.

23. Central Statistical Office [Zambia], Ministry of Health [Zambia], ICF International. Zambia Demographic and Health Survey 2013-2014. Lusaka, Zambia; 2014. 


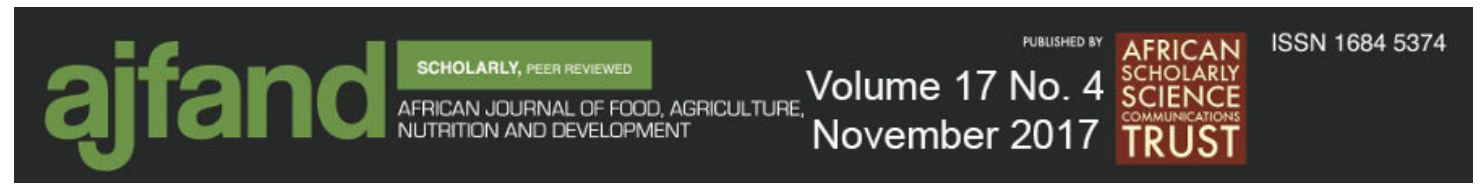

24. Wamani H, Astrom AN, Peterson S, Tumwine JK and T Tylleskär Boys are more stunted than girls in sub-Saharan Africa: a meta-analysis of 16 demographic and health surveys. BMC Pediatr. 2007; 7: 17.

25. Kung'u JK, Wright VJ, Haji HJ, Ramsan M, Goodman D, Tielsch JM, Bickle QD, Raynes JG and RJ Stoltzfus Adjusting for the Acute Phase Response Is Essential to Interpret Iron Status Indicators among Young Zanzibari Children Prone to Chronic Malaria and Helminth Infections. J Nutr. 2009; 139: 2124-31.

26. Siegel EH, Stoltzfus RJ, Khatry SK, Leclerq SC, Katz J and JM Tielsch Epidemiology of anemia among 4- to 17-month-old children living in south central Nepal. Eur J Clin Nutr. 2006; 60(2): 228-35.

27. Agho KE, Dibley MJ, D'Este C and R Gibberd Factors associated with haemoglobin concentration among Timor-Leste children aged 6-59 months. $J$ Health Popul Nutr. 2008; 26(2): 200-9.

28. Abubakar A, Uriyo J, Msuya SE, Swai M and B Stray-Pedersen Prevalence and risk factors for poor nutritional status among children in the Kilimanjaro region of Tanzania. Int J Environ Res Public Health. 2012; 9(10): 3506-18.

29. Souganidis ES, Sun K, de Pee S, Kraemer K, Rah JH, Moench-Pfanner R, Sari M, Bloem MW and RD Semba Relationship of maternal knowledge of anemia with maternal and child anemia and health-related behaviors targeted at anemia among families in Indonesia. Matern Child Health J. 2012; 16(9): $1913-25$.

30. Osório MM, Lira PIC and A Ashworth Factors associated with $\mathrm{Hb}$ concentration in children aged 6-59 months in the State of Pernambuco, Brazil. Br J Nutr. 2004; 91(2): 307-15.

31. Thurnham DI and CA Northrop-Clewes Infection and the etiology of anemia. In: Kraemer K, Zimmermann MB (eds.). Nutritional Anaemia. Basel, Switzerland: Sight and Life Press; 2007. p. 231-56.

32. Checkley W, Gilman RH, Black RE, Epstein LD, Cabrera L, Sterling CR and LH Mouton Effect of water and sanitation on childhood health in a poor Peruvian peri-urban community. Lancet. 2004; 363: 112-8.

33. Faber M Dietary intake and anthropometric status differ for anaemic and nonanaemic rural South African infants aged 6-12 months. J Health Popul Nutr. 2007; 25(3): 285-93. 


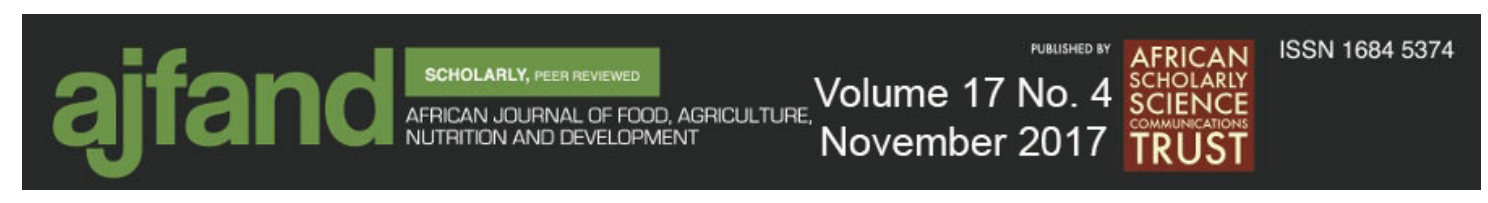

34. Hipgrave DB, Fu X, Zhou H, Jin Y, Wang X, Chang S, Scherpbier RW, Wang $Y$ and $S$ Guo Poor complementary feeding practices and high anaemia prevalence among infants and young children in rural central and western China. Eur J Clin Nutr. 2014; 68(8): 916-24.

35. Moursi MM, Arimond M, Dewey KG, Tre S, Ruel MT and F Delpeuch Dietary Diversity Is a Good Predictor of the Micronutrient Density of the Diet of 6- to 23-Month-Old Children in Madagascar. J Nutr. 2008; 138: 2448-53.

36. Northrop-Clewes $\mathbf{C}$ and DI Thurnham Biomarkers for the differentiation of anemia and their clinical usefulness. J Blood Med. 2013; 4: 11-22. 